(C) Copyright 1999 IEEE IEEE Vehicular Technology Conference (VTC'99), May 16-20, 1999, Houston, Texas, USA

Personal use of this material is permitted. However, permission to reprint/republish this material for advertising or promotional purposes or for creating new collective works for resale or redistribution to servers or lists, or to reuse any copyrighted component of this work in other works must be obtained from the IEEE. 


\title{
QUALITY OF SERVICE IN FUTURE EUROPEAN WIRELESS LANS
}

\author{
Martin Hagenauer \\ Institut für Nachrichtentechnik und Hochfrequenztechnik \\ Technische Universität Wien \\ Gußhausstraße 25/389, 1040 Wien, Austria \\ PH: +43-1-58801-38931 FAX: +43-1-5870583 \\ e-mail: Martin.Hagenauer@mobile.nt.tuwien.ac.at
}

\begin{abstract}
In a wireless LAN Quality of Service (QoS) is limited by hidden nodes, coverage and delay spread. The channel access mechanism of HIPERLAN Type 1 will provide a minimum collision rate in addition to a fair sharing of resources among all nodes. In this paper I have investigated the influence of the packet length, number of nodes and lifetime on the expected user data rate. The results show that a HIPERLAN Type 1 system is very robust under heavy load conditions. Earliest Data First (EDF)-scheduling of data packets enables tuning of multimedia performance by adding an optional buffer to the HIPERLAN user data interface.
\end{abstract}

\section{INTRODUCTION}

The European Telecommunications Standards Institute (ETSI) has set a new standard for high-speed wireless indoor data transfer between laptop computers [1]. HIPERLAN provides all services known from traditional wired Ethernet LANs via a standard PCMCIA card type III interface on a single radio channel. The frequency band between $5.15 \mathrm{GHz}$ and $5.30 \mathrm{GHz}$ will carry up to five channels with a maximum physical data rate of $23.54 \mathrm{Mbit} / \mathrm{s}$. As in GSM communications the Gaussian Minimum shift keying (GMSK) modulation with $\mathrm{BT}=0,3$ is used to achieve a good compromise between signal distortion in the receiver and spectral efficiency. HIPERLAN will set up "ad-hoc" networks between any nodes that are within the radio range of their modems. This requires a fully interconnected network and a low bit error rate.

In today's laptop computers an extra radio modem cannot make use of more than $2 \mathrm{~W}$ power without significantly reducing the recharging cycles of its batteries. The maximum allowed EIRP for a HIPERLAN Type 1 radio modem is $30 \mathrm{dBm}$ with a receiver sensitivity of $-70 \mathrm{dBm}$. To save power, prior signaling is done with FSK modulation and a lower bit rate of $1.47 \mathrm{Mbit} / \mathrm{s}$ and the equalizer switched off. The
HIPERLAN user interface provides a connectionless traffic channel on a basis of 'negotiating' Quality of Service (QoS) parameters using the Elimination-Yield Non-pre-emptive Priority Multiple Access (EY-NPMA) scheme.

\section{Channel Access Control}

The HIPERLAN channel access control (CAC) unit will provide fair channel access for all nodes within their radio range. This is achieved by eliminating as many packets as possible but not all from transmission. HIPERLAN uses the Elimination-Yield Non-preemptive Priority Multiple Access (EY-NPMA) scheme to provide channel access in combination with five internal priority levels. Figure 1 shows the sequence of contention and transmission phases in a synchronized channel access.

CAC priority levels determine the number of slots every node has to listen unless other nodes are already transmitting access bursts with higher priorities. The duration of each slot is $7,1 \mu \mathrm{s}$. Figure 2 shows the HIPERLAN Type 1 priority resolution after a synchronized channel access.

The transmission of access bursts is then used in the elimination part to further reduce the number of contending entities. All nodes with the longest access bursts survive for further transmission. The separation of nodes is done within slots of $9 \mu \mathrm{s}$. The probability for bursting in an individual slot is 0,5. Hence the probability for an elimination slot to have a total length of $n$ elimination slots is given by

$P_{E}(n)= \begin{cases}\frac{1}{2^{n+1}} & 0 \leq n<5 \\ 3,125 \% & n=5\end{cases}$ 
After this transmission the node listens for any contender with longer elimination bursts and defers from further transmission if one is detected. Again the listening cycle is used for a second contention among all surviving entities by a random delay of data transmission according to figure 3 . The nodes are separated within 0 to 9 slots of $7,1 \mu \mathrm{s}$ with equal likelihood.

If the radio channel is detected to be idle for $85 \mu \mathrm{s}$, unsynchronized data transmission may occur without any contention scheme. Upon detection of hidden nodes the channel access cycle is suspended for $500 \mathrm{~ms}$. An additional channel suspension interval is randomly selected between $1 \mathrm{~ms}$ and $5 \mathrm{~ms}$ for consecutive transmission attempts.

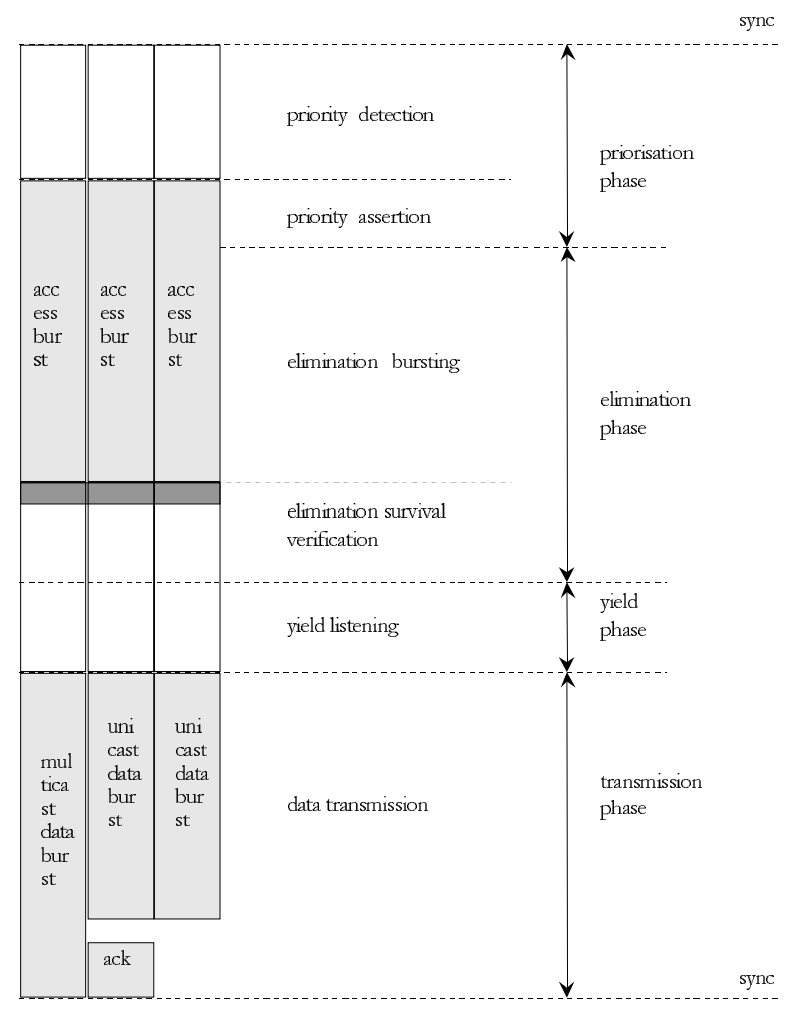

Figure 1: Synchronized HIPERLAN channel access

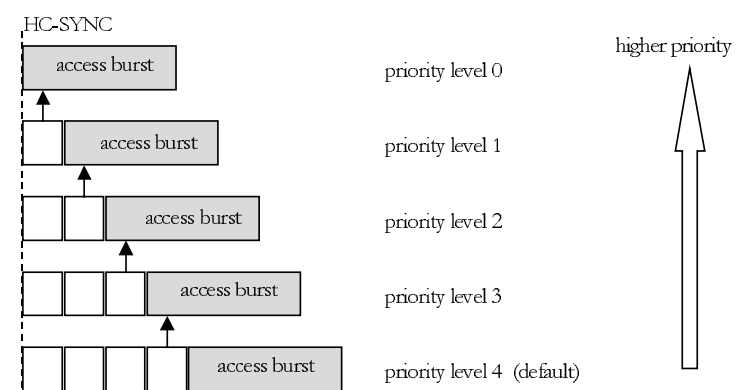

Figure 2: HIPERLAN Type 1 priority resolution

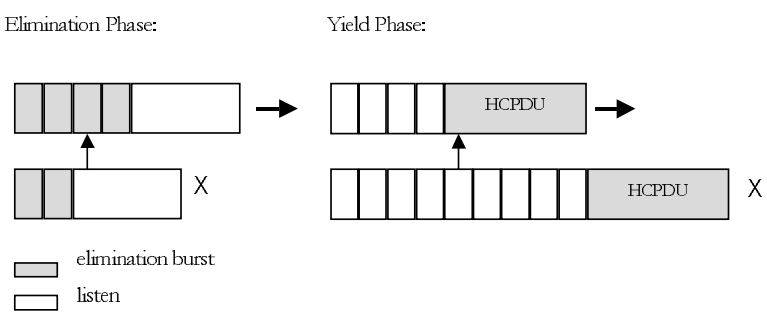

Figure 3: HIPERLAN Type 1 node selection

\section{Medium Access Control}

Every HIPERLAN physical channel can carry several virtual channels with different nodes participating in different virtual channels. Within each virtual channel various services can be active at the same time. A HIPERLAN node therefore has to make an internal selection of packets to transmit. The decision is based upon the QoS parameters supplied by the user (lifetime of packets, user priority) and an internal mapping of pending data packets to the CAC transmit priorities according to figure 4 .

The user data transfer function of the HIPERLAN medium access control (MAC) sublayer calculates each packets Normalized Residual MAC Lifetime (NRML) parameter. The NRML is defined as the remaining lifetime of a packet normalized to the remaining number of hops to its target destination.

$\mathrm{NRML}=\frac{\text { MSDU lifetime }- \text { elapsed time since MSDU is accepted }}{\text { remaining number of hops in routing packet }}$

Normalized residual lifetimes below $80 \mathrm{~ms}$ result in privileged transmission for packets with high user priority.

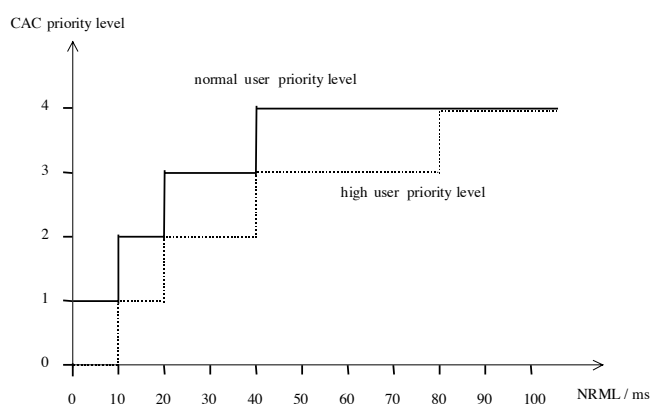

Figure 4: HIPERLAN Type 1 internal packet selection 
The selection of packets for further transmission follows the rules:

1) select the HIPERLAN MAC Protocol Data Units (HMPDU) with the highest channel access priority (i.e. lowest CAC priority level)

2) from which select the HMPDU with the shortest normalized residual lifetime

3) from which select anyone.

\section{SIMULATION RESULTS}

Several simulations have been made to evaluate the performance of the HIPERLAN channel access mechanism (CAM) with respect to its Quality of Service parameters. The data rate was computed at the upper boundary of the HIPERLAN CAC-sublayer to provide results seen from a higher-layer point of view. Every contending node has its transmission buffer permanently active and power saving deactivated. Undisturbed radio transmission and a fully connected network were assumed. Since routing algorithms are not part of the current HIPERLAN system definition, but can be implemented on higher layers, I did not include any forwarding of data packets.

Figure 5 shows the total throughput for a connectionless file transfer as a function of the packet length. The graph clearly shows the highest data rates for maximum size packets with 47 data blocks due to the smaller relative overhead of signaling and synchronization. The data rate falls below half of its maximum value for very short packets with less than 6 data blocks only.

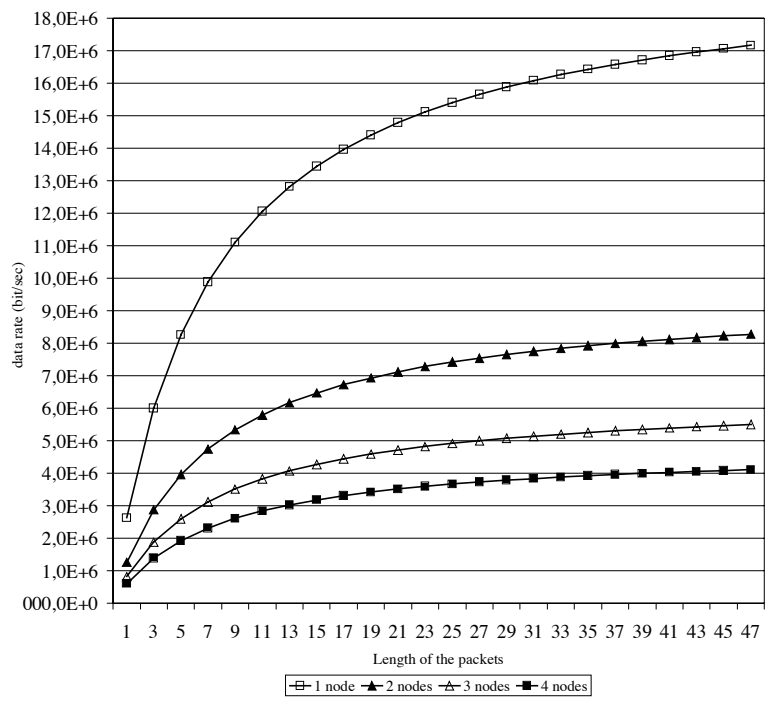

Figure 5: HIPERLAN data rate vs. length of packets

Figures 6 and 7 show the user data rate with respect to the number of contending nodes. The data rate decreases with an increasing number of competitors due to a fair sharing of resources. One can see that a single HIPERLAN physical channel is able to carry up to 8 virtual channels of $2 \mathrm{Mbit} / \mathrm{s}$ or 240 virtual channels of $64 \mathrm{kbit} / \mathrm{s}$ simultaneously.

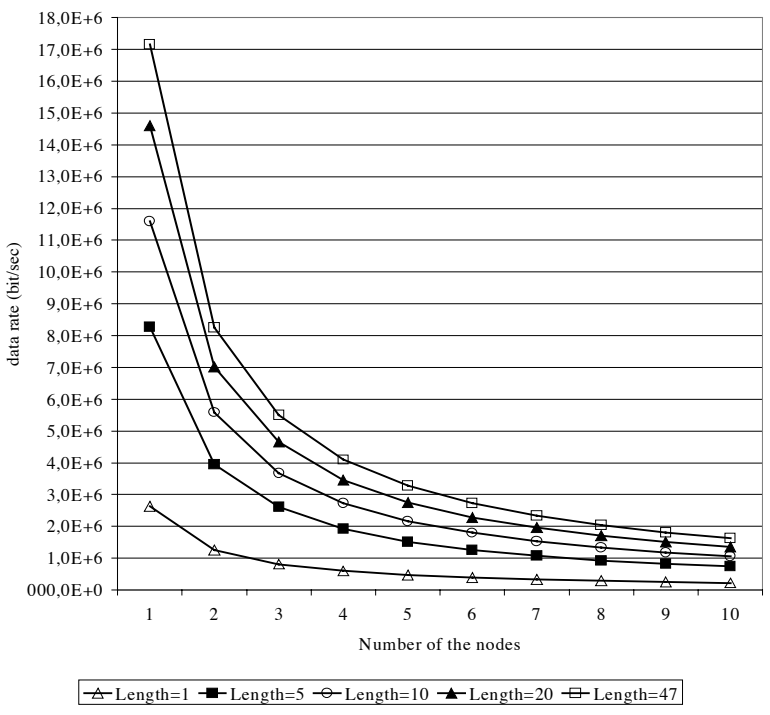

Figure 6: HIPERLAN data rate vs. number of nodes, $2 \mathrm{Mbit} / \mathrm{s}$

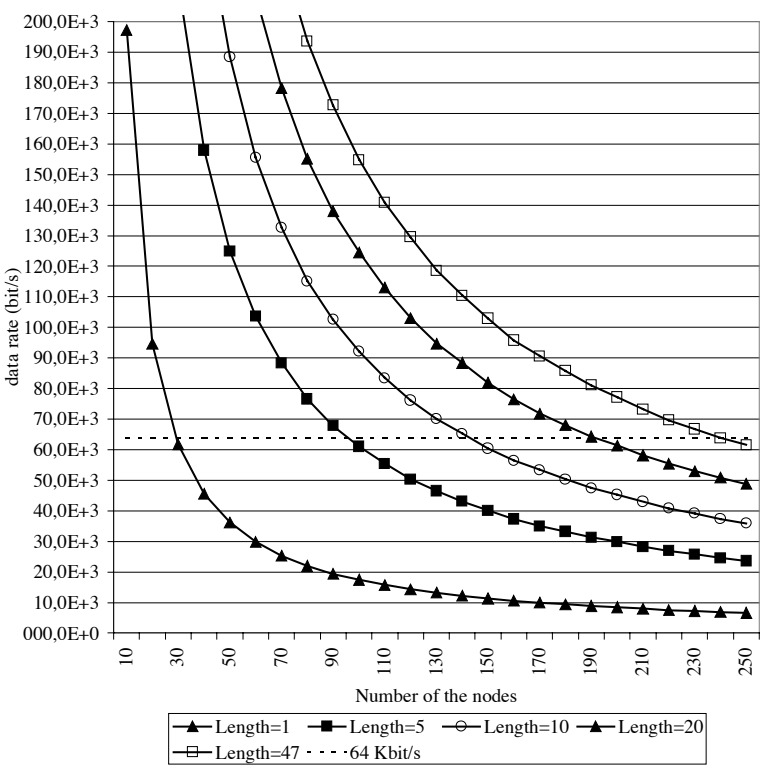

Figure 7: HIPRELAN data rate vs. number of nodes, $64 \mathrm{kbit} / \mathrm{s}$

Data packets are passed to the HIPERLAN CAMsublayers with an initial lifetime QoS parameter. The lifetime of each packet is reduced during queuing and processing of data. When the lifetime of a packet has elapsed, the data is withdrawn from any further transmission. The HIPERLAN EDF-queuing scheme defines four levels $s_{i}$ that shift earlier packets towards 
lower CAC priority levels for privileged transmission. Figure 8 shows the influence of this EDF-queuing on the data rate in a scenario with three nodes simultaneously transmitting packets of maximum length. The transition from normal file transfer traffic with higher lifetimes of packets towards more urgent multimedia traffic shows peaks with slightly increased data rates. The maximum collision rate stays well below $4 \%$. In figure 9 one can see that the collision rate drops to very low values within the length of one packet, because the EDF-limit then selects only one packet for privileged data transmission, if the channel access mechanism is fair. The position of these points with minimum collision rate is a function of the packet length and number of contending nodes. If all HIPERLAN nodes use the same packet length one can estimate the lifetime providing a minimum collision rate with

$$
\mathrm{m}_{\mathrm{i}}=\mathrm{s}_{\mathrm{i}}+(\mathrm{n}-1) \cdot \mathrm{p}
$$

where $\mathrm{s}_{\mathrm{i}}$ takes the values $\{10 \mathrm{~ms}, 20 \mathrm{~ms}, 40 \mathrm{~ms}\}$ for low user priority and $\{10 \mathrm{~ms}, 20 \mathrm{~ms}, 40 \mathrm{~ms}, 80 \mathrm{~ms}\}$ for high user priority, $\mathrm{n}$ is the number of nodes and $\mathrm{p}$ denotes the transmission time for one packet. Further reduction of the lifetime again increases the collision rate in several steps according to the probability of having two, three ore more nodes transmitting with the next lower CAC priority level. The user data rate is reduced accordingly. The influence of adding an optional FIFO buffer to the HIPERLAN medium access control user interface is shown in figure 10. Packet lifetime normally is in the range of $20 \mathrm{~ms}$ to $200 \mathrm{~ms}$ for multimedia traffic and from $500 \mathrm{~ms}$ to $1000 \mathrm{~ms}$ for file transfer traffic. Note that the user data rate for multimedia traffic of 8 nodes can be kept above the UMTS data rate of $2 \mathrm{Mbit} / \mathrm{s}$ by adding a buffer for 10 packets, when the residual packet error rate is better than $1 \%$. Longer buffer sizes can be of advantage when normal file transfer traffic is used. However, one has to consider that the transmission time for any packets through the FIFO buffer must not exceed its initial supplied lifetime.

Assuming the instantaneous number of transmitting nodes is known, every HIPERLAN node then can adapt its buffer length to minimize the collision rate on the physical radio channel. Since the transmission buffer of a HIPERLAN node might not always be activated, one needs a traffic model for an estimation of the actual number of transmitters. The parameters for this traffic model can either be obtained by adaptation techniques or are published by the sending HIPERLAN entities.

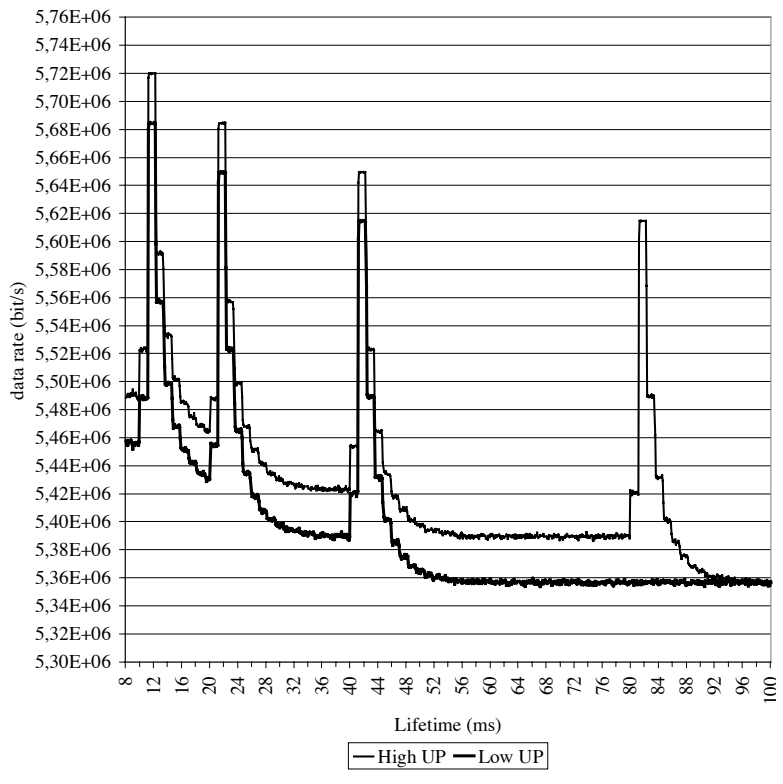

Figure 8: HIPERLAN data rate vs. lifetime of packets, 3 nodes, no buffer

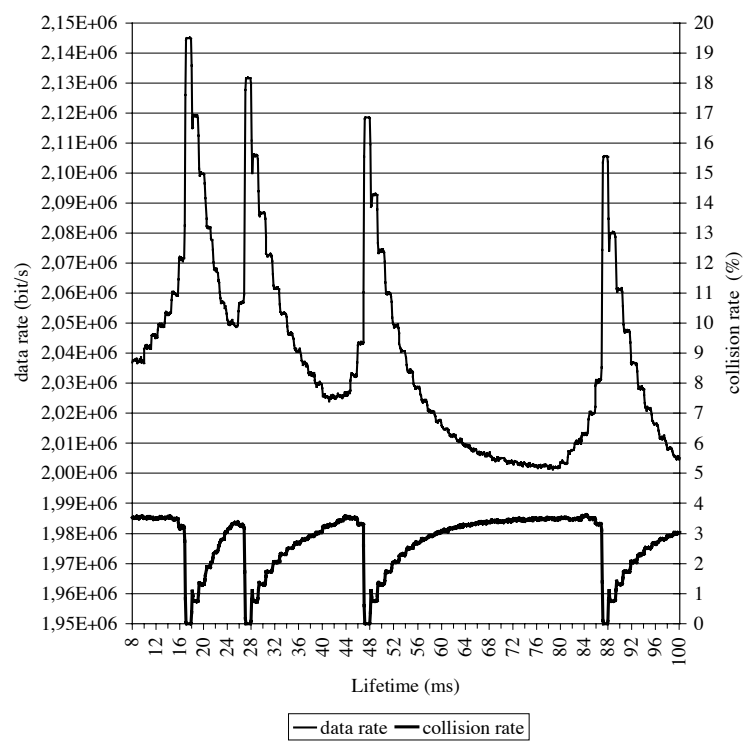

Figure 9: HIPERLAN data rate and collision rate vs. lifetime of packets, 8 nodes, no buffer 


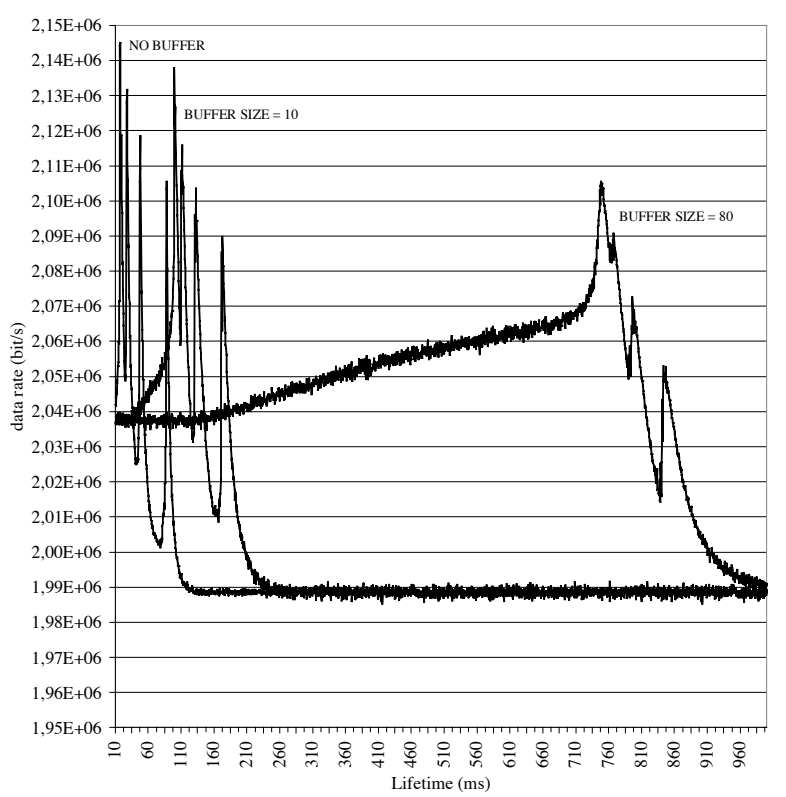

Figure 10: Influence of buffers on HIPERLAN throughput with 8 nodes

\section{CONCLUSIONS}

The HIPERLAN user data rate has been simulated with respect to its packet length, number of nodes and lifetime. It is shown that a HIPERLAN physical channel can carry up to 8 virtual traffic channels of $2 \mathrm{Mbit} / \mathrm{s}$ simultaneously with a fair channel access. The collision rate was found to disappear completely for certain user provided lifetimes of the packets. Adding an optional buffer to the HIPERLAN MAC user interface can shift the position of these points.

\section{REFERENCES}

(1) ETSI, "Broadband Radio Access Networks (BRAN); HIgh PErformance Radio Local Area Network (HIPERLAN); Type 1; Functional specification"; EN 300652 V1.2.1, 1998-07

(2) ETSI, "Radio Equipment and Systems (RES); HIgh PErformance Radio Local Area Networks (HIPERLAN); Requirements and architectures for Wireless ATM Access and Interconnection", TR 101 031 V1.1.1, 1997-07

(3) ETSI, "Radio Equipment and Systems (RES); HIgh PErformance Radio Local Area Networks (HIPERLAN); Architecture for Time Bound Services (TBS)", ETR 226, 1995-10

(4) CEPT Recommendation T/R 22-06, Relating to the harmonised frequency bands for high performance radio local area networks",1992

(5) S. Chevrel, A. H. Aghvami, H. Y. Lach, L. Taylor, Analysis and optimisation of the HIPERLAN Channel Access Contention Scheme", ireless Personal Communications, Vol. 4, No. 1, 1997

(6) K. Fu, Y. J. Guo, S. K. Barton, "Performance of the EY-NPMA Protocol", Wireless Personal Communications, Vol. 4, No. 1, 1997

(7) P. Jacquet, P. Minet, P. Mühlethaler, N. Rivierre, "Priority and Collision Detection with Active Signaling - The Channel Access Mechanism of HIPERLAN", Wireless Personal Communications, Vol. 4, No. 1, 1997 